\title{
Branchiogenic carcinoma with high-risk-type human papillomavirus infection: A case report
}

\author{
HIROYUKI MAEDA $^{1}$, ZEYI DENG $^{1,2}$, TARO IKEGAMI $^{1}$, SEN MATAYOSHI $^{1}$, SHINYA AGENA $^{1}$, \\ ASANORI KIYUNA $^{1}$, YUKASHI YAMASHITA ${ }^{1}$, TAKAYUKI UEHARA ${ }^{1}$, AKIRA GANAHA $^{1}$ and MIKIO SUZUKI ${ }^{1}$ \\ ${ }^{1}$ Department of Otorhinolaryngology, Head and Neck Surgery, Graduate School of Medicine, University of the Ryukyus, \\ Nishihara, Okinawa 903-0215, Japan; ${ }^{2}$ Department of Otorhinolaryngology, Head and Neck Surgery, \\ Zhujiang Hospital, Southern Medical University, Guangzhou, Guangdong 510280, P.R. China
}

Received March 28, 2015; Accepted June 29, 2016

DOI: $10.3892 / 01.2016 .4907$

\begin{abstract}
Branchiogenic carcinoma (BC) usually appears as a mass lesion with a predominant cystic component. Since lymph node metastasis from oropharyngeal carcinoma (OPC) has a cystic appearance, it is occasionally difficult to distinguish between BC and nodal metastases from clinically silent OPC. Factors associated with the malignant transformation process in $\mathrm{BC}$ remain obscure. The present study reports the case of a 56-year-old man with a right cystic cervical mass that was diagnosed as squamous cell carcinoma based on examination by fine-needle aspiration biopsy. The primary tumor could not be detected despite several imaging examinations, a pan-endoscopy of the head and neck, esophagus and stomach, biopsies of the head and neck regions, and bilateral tonsillectomies. The pathological findings of the surgical specimens from a radical neck dissection were consistent with the histological characteristics of $\mathrm{BC}$, with evidence of transition from dysplasia through intraepithelial carcinoma to invasive carcinoma. Normal squamous epithelium and dysplastic and cancerous portions in the BC showed strong p16 ${ }^{\mathrm{INK} 4 \mathrm{a}}$ immunoreactivity. The expression of $\mathrm{p} 16^{\mathrm{INK} 4 \mathrm{a}}$ was also observed in all 9 nodal metastases in the neck dissection specimens. The cystic formation observed in the BC was not observed in the nodal metastases. As the presence of human papillomavirus-16 in the tumor was confirmed by polymerase chain reaction, quantitative polymerase chain reaction was
\end{abstract}

Correspondence to: Professor Mikio Suzuki, Department of Otorhinolaryngology, Head and Neck Surgery, Graduate School of Medicine, University of the Ryukyus, 207 Uehara, Nishihara, Okinawa 903-0215, Japan

E-mail:suzuki@med.u-ryukyu.ac.jp

Abbreviations: $\mathrm{BC}$, branchiogenic carcinoma; CT, computed tomography; FDG, ${ }^{18}$ F-fluorodeoxyglucose; HPV, human papillomavirus; OPC, oropharyngeal carcinoma; PCR, polymerase chain reaction; SCM, sternocleidomastoid

Key words: branchiogenic carcinoma, human papillomavirus, high-risk-type, integration, $\mathrm{p} 16^{\mathrm{INK} 4 \mathrm{a}}$ expression employed for the measurement of human papillomavirus-16 viral load and integration. The results showed that the viral load of human papillomavirus-16 was $3.01 \times 10^{7} / 50 \mathrm{ng}$ genomic DNA, and the E2/E6 ratio was 0.13 , so the integration state was judged to be the mixed type. To the best of our knowledge, this is the first report of $\mathrm{BC}$ associated with high-risk-type human papillomavirus infection. The study indicates that a human papillomavirus-positive neck mass may not necessarily be OPC, but that it could be $\mathrm{BC}$ with a poor prognosis. This report lends support to the existence of $\mathrm{BC}$ and proposes that the etiology is human papillomavirus infection.

\section{Introduction}

Branchiogenic carcinoma (BC) is an extremely rare malignant neck tumor that usually appears as a mass lesion with a prominent cystic component. Since oropharyngeal carcinoma (OPC) metastasis in the lymph nodes also has a cystic appearance, it is occasionally difficult to distinguish between $\mathrm{BC}$ and metastases in the cervical lymph nodes from clinically silent OPC.

Martin et al (1) proposed the following criteria for the diagnosis of $\mathrm{BC}$, stating the fourth criterion as the most important for confirming BC. First, the cervical tumor must occur at a point along an imaginary line that extends between just anterior to the tragus of the ear and downward along the anterior border of the sternocleidomastoid (SCM) muscle to the clavicle. Second, the histological appearance of the mass should be consistent with an origin from tissue known to be present in the branchial vestigia. Third, a survival period of at least 5 years, with periodic examinations, must be recorded without the development of any other lesion that could be the primary tumor. Fourth, histology should demonstrate the presence of cancer in the wall of an epithelial cyst situated in the lateral aspect of the neck. Khafif et al disagreed with the third criterion, as patients may succumb to unrelated causes prior to the 5-year milestone (2). In addition, a number of patients receive post-operative irradiation, which may result in control of an occult primary tumor. Therefore, Khafif et al (2) proposed replacement of this criterion with two additional criteria: i) The absence of an identifiable primary cancer in another location, as determined by a thorough evaluation, including appropriate biopsies; and ii) the clear histological 

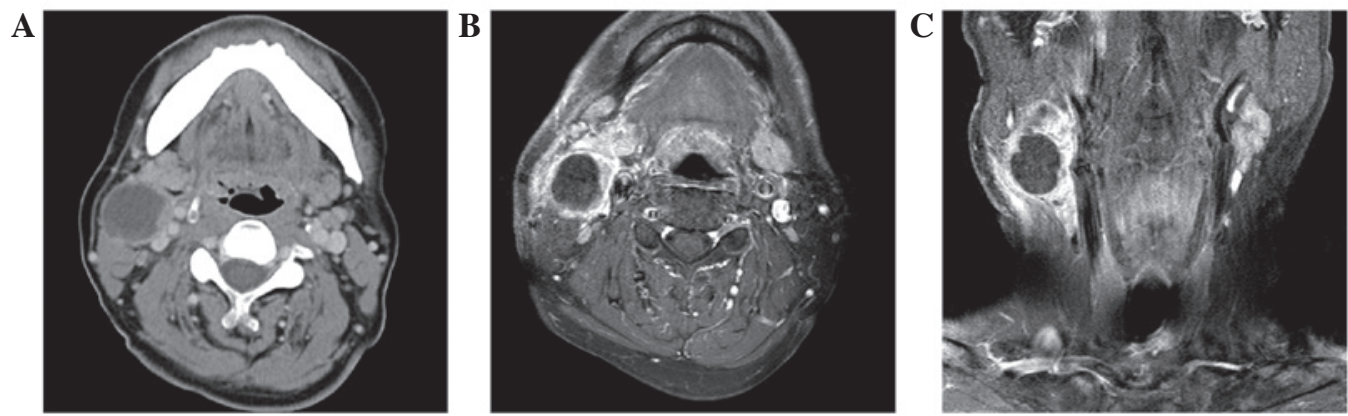

Figure 1. Computed tomography (CT) and magnetic resonance imaging of the neck. (A) CT with contrast enhancement. The tumor was anterior to the sternocleidomastoid muscle. The tumor contained a prominent cystic portion and a solid portion. (B) T1-weighted image with gadolinium enhancement (axial view). The cyst wall and solid portion (medial region of the tumor) showed high signal intensity on magnetic resonance imaging. (C) T1-weighted image with gadolinium enhancement (coronal view). The solid portion was located at the superiomedial aspect of the tumor.

identification of a cystic structure that is partially lined by normal squamous or pseudostratified columnar epithelium, with a gradual transition through intraepithelial carcinoma to invasive squamous cell carcinoma. This proposal represents the most recent criteria for the diagnosis of $\mathrm{BC}$.

The present study describes a case of BC that fulfilled the criteria of Khafif et al (2) and was possibly caused by high-risk-type human papillomavirus (HPV) infection. Written informed consent was obtained from the patient for publication of this case report and any accompanying images. This report adhered to the tenets of the Declaration of Helsinki.

\section{Case report}

Patient data. A 56-year-old man, who was a non-smoker with diabetes mellitus, presented to a referral hospital in 2012 with a 4-month history of a non-pulsatile mass on the right side of the neck. At that time, no sore throat, fever, weight loss, cough or otalgia were reported. The patient's blood sugar level was well controlled with medication and the family history was unremarkable. A physical examination had revealed a 6-cm non-tender mass, and computed tomography (CT) and magnetic resonance imaging scans showed a $3.6 \times 5.0-\mathrm{cm}$, predominantly cystic mass, with a peripheral solid component. The mass was located posterior to the submandibular gland and anteromedial to the SCM muscle (Fig. 1). Several small lymph nodes in the right neck were also detected on CT.

The patient was referred to the University Hospital, University of the Ryukyus (Nishihara, Japan) for treatment of a right neck lesion, 1 month subsequently to the intial visit to the aforementioned hospital. Ultrasound-guided fine-needle aspiration biopsy was reported as class V (squamous cell carcinoma). No tumor was identified on endoscopic examination of the head and neck, esophagus and stomach, or on thoracoabdominal CT. Positron emission tomography/CT showed ${ }^{18} \mathrm{~F}$-fluorodeoxyglucose (FDG) uptake in the solid portion and cyst wall of the tumor (Fig. 2). Squamous cell carcinoma antigen in the serum, a tumor marker for SCC, was slightly elevated at $1.9 \mathrm{ng} / \mathrm{ml}$ (normal, $\leq 1.5 \mathrm{ng} / \mathrm{ml}$ ). No tumor was identified in random biopsies of the nasopharynx, tongue base, bilateral palatine tonsils, larynx and hypopharynx. A bilateral tonsillectomy was also performed to exclude the possibility of lymph node metastasis from an extremely small tonsillar carcinoma, but no cancerous lesion was noted on examination of serial sections of the tonsils.
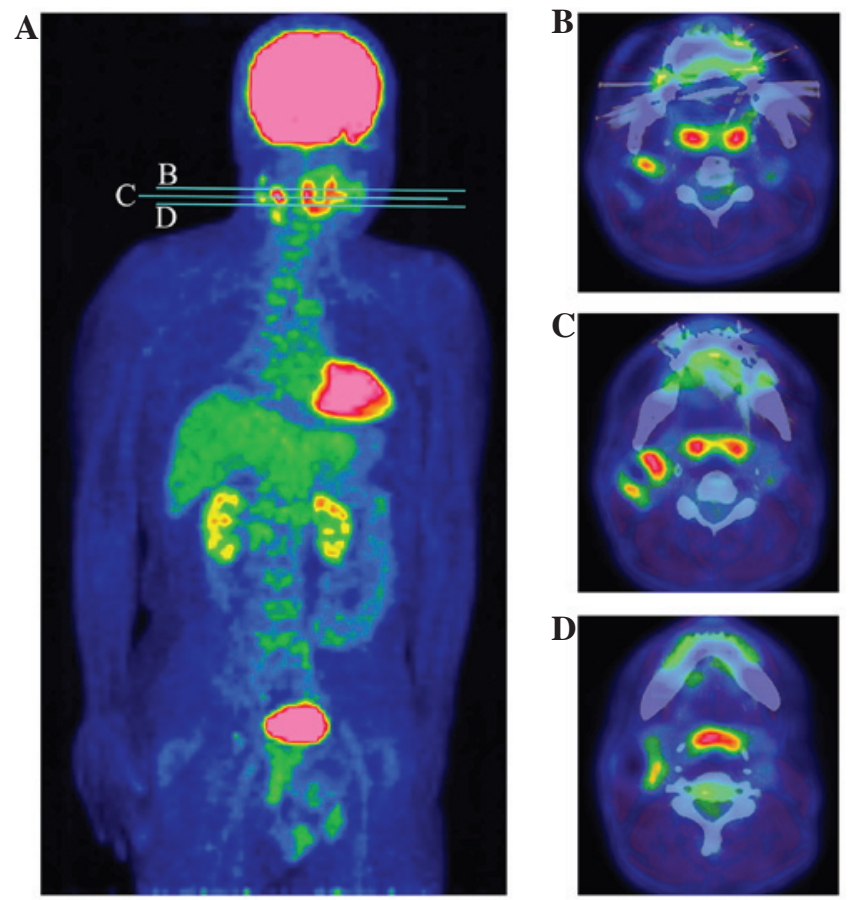

Figure 2. Images of ${ }^{18} \mathrm{~F}$-fluorodeoxyglucose (FDG) positron emission tomography combined with computed tomography. (A) Whole-body scan FDG uptake was observed in the right cervical mass (maximum standardized uptake value, 9.2). Physiological FDG uptake was also observed in the brain, tonsils, heart, kidneys and urinary bladder. (B) Axial slice at level B in (A). (C) Axial slice at level C in (A). (D) Axial slice at level D in (A). FDG uptake was observed at the cyst wall and in the solid region of the tumor. The palatine tonsil and tongue base also exhibited physiological FDG uptake.

From the aforementioned history and findings, a preliminary diagnosis of cervical lymph node metastasis with an unknown primary site was formed, and a right neck dissection was performed. Since the tumor adhered to the internal jugular vein and SCM muscle, a radical neck dissection was performed. Intraoperatively, several enlarged lymph nodes were noted around the tumor. Pathological examination revealed a cystic mass containing poorly-differentiated, invasive squamous cell carcinoma (Fig. 3A), with focal areas of normal squamous epithelium and mild to severe dysplasia (Fig. 3B). In particular, there was transition from dysplasia (Fig. 4A and B) through intraepithelial carcinoma to invasive carcinoma with pleomorphism and multiple mitoses (Fig. 4A and C). Koilocytosis was 

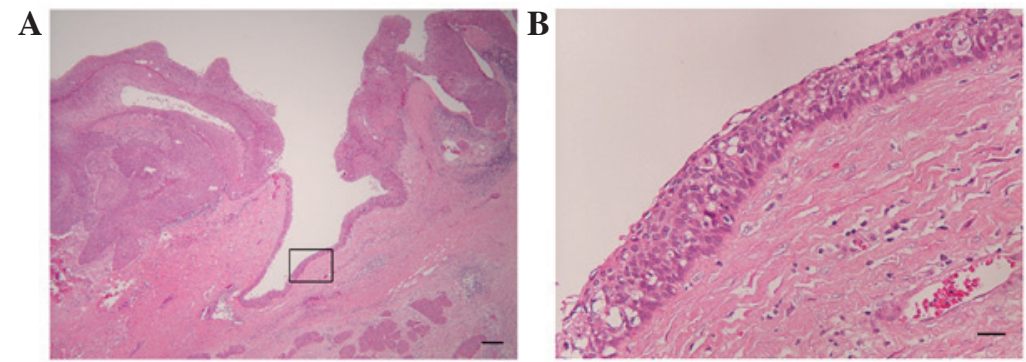

Figure 3. Hematoxylin-eosin staining of the tumor. (A) Low magnification view of the tumor. The tumor formed a cyst consisting of normal squamous epithelium and invasive cancer. Scattered lymphoid cells were observed in the subepithelial layer. Bar, $100 \mu \mathrm{m}$. (B) High magnification view of the boxed region in (A), showing squamous epithelium with a few areas of dysplasia. The epithelial cells showed marked koilocytosis. Bar, $25 \mu \mathrm{m}$.

A

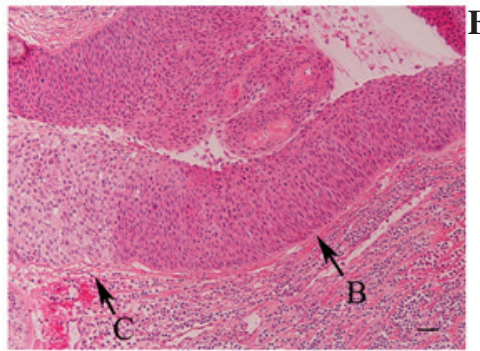

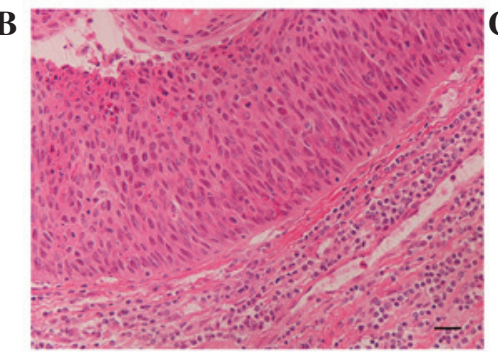

Figure 4. Transition from severe dysplasia to squamous cell carcinoma (hematoxylin-eosin staining). (A) Low magnification view of the transition area. Highly dysplastic epithelium (arrow B) with a change to squamous cell carcinoma (arrow C). Bar, $100 \mu \mathrm{m}$. (B) High magnification view of the region indicated by arrow B in (A). The epithelium has retained a distinct basal cell layer and cellular differentiation. Epithelial cell atypia was minimal. Bar, $25 \mu$ m. (C) High magnification view of the region indicated by arrow $\mathrm{C}$ in (A). The epithelium has lost cellular differentiation and polarity. Bar, $25 \mu \mathrm{m}$.
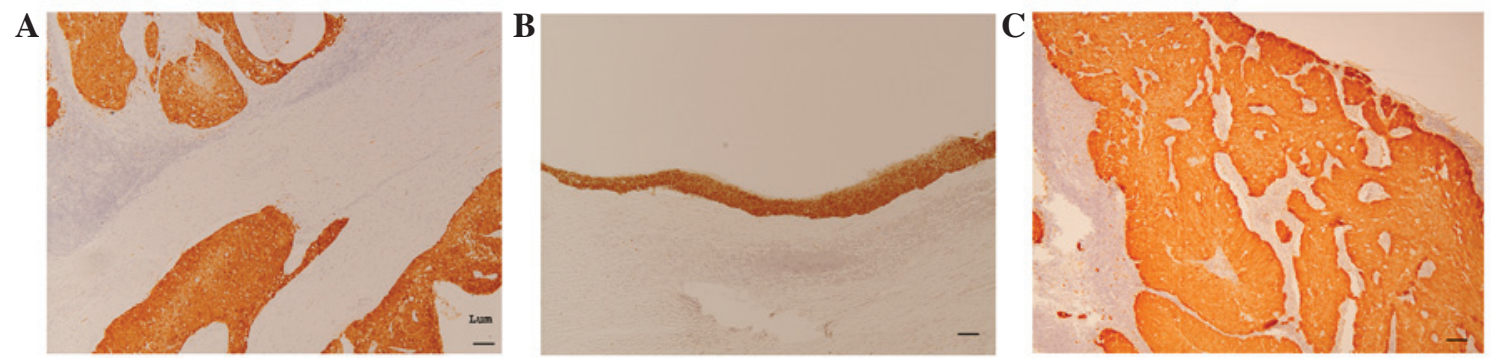

Figure 5. Expression of p16 ${ }^{\mathrm{INK} 4 \mathrm{a}}$. (A) The solid portion of the tumor. The tumor cells showed strong p16 ${ }^{\mathrm{INK} 4 \mathrm{a}}$ expression. Bar, $100 \mu \mathrm{m}$. (B) The squamous epithelium also showed strong $\mathrm{p} 16^{\mathrm{INK} 4 \mathrm{a}}$ expression. Bar, $100 \mu \mathrm{m}$. (C) Nodal metastasis. Tumor cells in the lymph node showed strong p16 $6^{\mathrm{INK} 4 \mathrm{a}}$ expression. There was no cystic formation in the nodal metastasis. Bar, $100 \mu \mathrm{m}$. Lum, lumen of the tumor.

present in the areas of dysplasia and carcinoma. An examination of the lymph nodes revealed 3 lymph node metastases at level IIb, 5 at level III and 1 at level IV, and none of the nodal metastases exhibited a cystic formation as was observed in the main tumor. The surgical margins were negative.

Considering the clinical history, and the imaging and histological findings, the tumor was diagnosed as BC, fulfilling the diagnostic criteria proposed by Khafif et al (2). Since lymph node metastases were noted, concurrent chemoradiotherapy was administered with 50.4 Gy irradiation to the entire neck, including the oropharynx, and two cycles of nedaplatin plus 5-fluorouracil (3). Multiple lung metastases appeared at 6 months post-surgery, followed by the occurrence of brain metastasis, despite several courses of docetaxel with nedaplatin plus 5-fluorouracil (3). The patient succumbed 1 year after surgery. No primary tumor or no lymph node metastasis in the head and neck were noted at the time of mortality.
Immunohistochemical examination of p16 $6^{\text {INK4a }}$ expression. The expression of $\mathrm{p} 16^{\mathrm{INK} 4 \mathrm{a}}$ was investigated in the primary lesion, nodal metastases and bilateral palatine tonsils, following the methods described in our previous study (4). Strong $\mathrm{p} 16^{\mathrm{INK} 4 \mathrm{a}}$ expression was observed in the squamous epithelium, dysplastic epithelium and carcinoma within the BC (Fig. 5A and B). Notably, the nodal metastases also exhibited strong p16 ${ }^{\mathrm{INK} 4 \mathrm{a}}$ expression (Fig. 5C). No expression was observed in either palatine tonsil.

HPV infection, viral load and integration status. A fresh specimen of BC was tested for the presence of HPV, the viral load and the integration status, following the methods described in our previous studies $(5,6)$. Polymerase chain reaction (PCR) for HPV using a consensus primer was positive (Fig. 6). Direct sequencing of the PCR product revealed the presence of HPV-16 infection. Quantitative PCR showed that the viral 
A

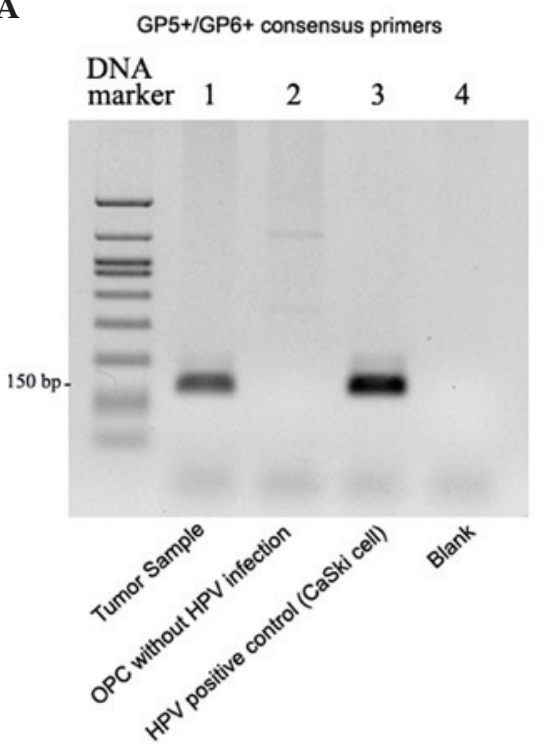

B

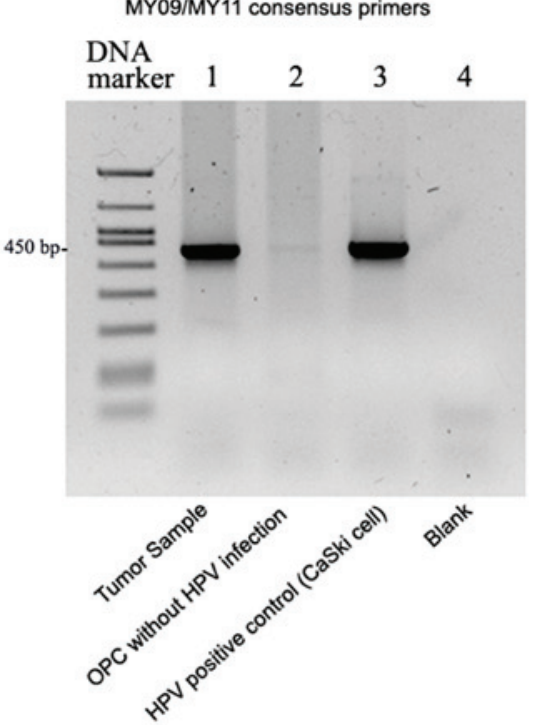

C

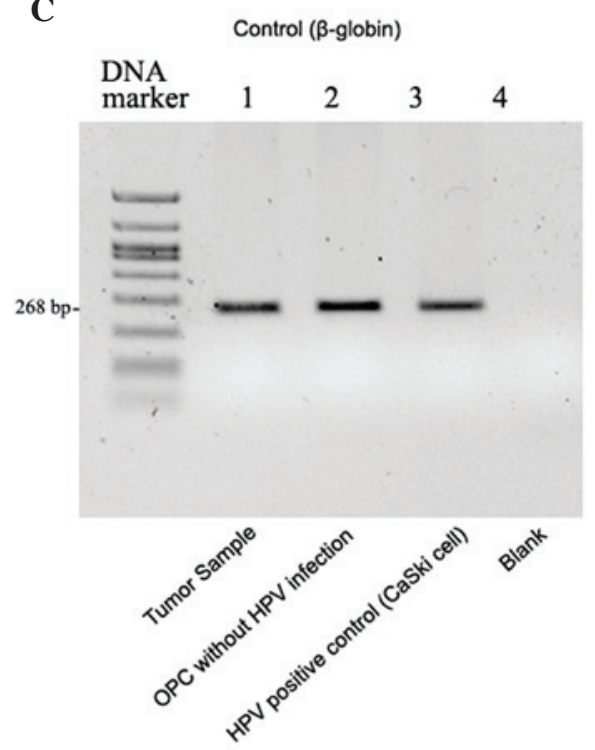

Figure 6. Polymerase chain reaction (PCR) for detection of human papilloma virus (HPV). (A) PCR results using GP5 ${ }^{+} / \mathrm{GP} 6^{+}$consensus primer sets $(6)$. (B) PCR results using MY09/MY11 consensus primer sets (6). (C) PCR results of the internal control, $\beta$-globin (6). Lane 1, tumor sample; lane 2, oropharyngeal cancer sample without HPV infection as the negative control; lane 3, CaSki cells as the positive control; and lane 4, no sample DNA in the reaction solution. The expression of HPV DNA was observed in the tumor sample and direct sequencing of the PCR product indicated HPV-16.

load of HPV-16 was 3.01 x107/50 ng genomic DNA and that the E2/E6 ratio was 0.13 (6); thus, the HPV-16 integration status was judged to be the mixed type.

\section{Discussion}

$\mathrm{BC}$, an extremely rare primary tumor arising from the remnant of the branchial pouches, is a rare tumor that was first reported by Von Volkmann in 1882 (7). However, BC has been considered a distinct entity only in the past few decades. Since metastasis from OPC in the lymph nodes frequently exhibits cystic formation, similar to that observed in $\mathrm{BC}$, recent reports have indicated that the majority of cystic lesions previously diagnosed as squamous cell carcinoma were lymph node metastases from OPC, particularly tonsillar carcinoma (8-10). Yasui et al (10) reported that an occult HPV-positive primary tumor will most likely occur in the oropharynx and that determining the HPV status of fine-needle aspirates facilitates the diagnosis of cystic node metastasis.

The present case matched the criteria proposed by Khafif et al (2), but did not match those proposed by Martin et al (1), as the patient succumbed to distant metastasis within a 5-year period. Since normal squamous epithelium at the cyst wall and transition from dysplasia to carcinoma were observed, the case was judged to be a typical BC.

There was evidence of high-risk-type HPV infection and integration in the tumor, with a high viral load. In a previous study (6), the viral load in tonsillar carcinoma with HPV-16 infection ranged from $1.54 \times 10^{2}-1.34 \times 10^{7} / 50 \mathrm{ng}$ genomic DNA (median, $4.13 \times 10^{5} \mathrm{ng}$ ). Compared with the viral load in tonsillar carcinoma with HPV infection, the viral load in the present case was quite high. To determine the physical status of HPV-16, the present study applied quantitative PCR, as described in our previous study (6). Tonsillar carcinoma with high-risk-type HPV infection usually shows HPV integration (6), which suggests that malignant transformation of branchiogenic cyst may arise from high-risk-type HPV infection and integration. To the best of our knowledge, the present study is the first report in the English literature to demonstrate a close association between BC and HPV infection. The marked koilocytosis and $\mathrm{p} 16^{\mathrm{INK} 4 \mathrm{a}}$ expression in the squamous epithelium, dysplastic epithelium and cancerous areas support this assumption.

The prognosis of patients with OPC associated with HPV infection is better than that for those individuals without HPV infection (11). The treatment regimen for OPC associated with HPV infection that was used in the present results in a 5-year overall survival rate of $>90 \%(3,5)$. Despite the presence of HPC infection, the present case had a disease course that was different from OPC associated with HPV infection. This suggests that BC associated with HPV infection may have different clinical characteristics compared with OPC associated with HPV infection.

It remains unclear why high-risk-type HPV infects the branchiogenic cyst or carcinoma. One possible explanation is that HPV infects the palatine tonsils and enters the branchial cyst though the remnant or a patent communicating route. However, the present study was unable to identify a communication between the oropharyngeal cavity and $\mathrm{BC}$, as a tonsillectomy was performed prior to the neck dissection. The aforementioned experimental data suggest that persistent HPV infection in the cyst causes HPV integration and malignant transformation. However, further studies are required to confirm this.

In conclusion, this is the first report of $\mathrm{BC}$ associated with high-risk-type HPV infection. Although there may be fewer BC than cystic lymph node metastases from OPC, a HPV-positive neck mass is not necessarily associated with OPC, but can also occur with $\mathrm{BC}$ that has a poor prognosis. This study provides further evidence of the existence of $\mathrm{BC}$ and proposes that the cause is HPV infection. 


\section{Acknowledgements}

This study was supported by grants KAKENHI 23592534, KAKENHI 26462610 and KAKENHI 26462611 awarded by the Japan Society for the Promotion of Science. The authors would like to thank the Ryukyu Society for the Promotion of Oto-Rhino-Laryngology for proving editorial assistance and technical assistance.

\section{References}

1. Martin H, Morfit HM and Ehrlich H: The case for branchiogenic cancer (malignant branchioma). Ann Surg 132: 867-887, 1950.

2. Khafif RA, Prichep R and Minkowitz S: Primary branchiogenic carcinoma. Head Neck 11: 153-163, 1989.

3. Hasegawa M, Maeda H, Deng Z, Kiyuna A, Ganaha A, Yamashita Y, Matayoshi S, Agena S, Toita T, Uehara T and Suzuki M: Prediction of concurrent chemoradiotherapy outcome in advanced oropharyngeal cancer. Int J Oncol 45: 1017-1026, 2014.

4. Deng Z, Hasegawa M, Aoki K, Matayoshi S, Kiyuna A, Yamashita Y, Uehara T, Agena S, Maeda H, Xie M and Suzuki M: A comprehensive evaluation of human papillomavirus positive status and p16INK4a overexpression as a prognostic biomarker in head and neck squamous cell carcinoma. Int J Oncol 45: 67-76, 2014.

5. Deng Z, Hasegawa M, Yamashita Y, Matayoshi S, Kiyuna A, Agena S, Uehara T, Maeda H and Suzuki M: Prognostic value of human papillomavirus and squamous cell carcinoma antigen in head and neck squamous cell carcinoma. Cancer Sci 103 2127-2134, 2012
6. Deng Z, Hasegawa M, Kiyuna A, Matayoshi S, Uehara T, Agena S, Yamashita Y, Ogawa K, Maeda H and Suzuki M: Viral load, physical status, and E6/E7 mRNA expression of human papillomavirus in head and neck squamous cell carcinoma. Head Neck 35: 800-808, 2013.

7. Von Volkmann R: Deep branchiogenic neck carcinoma. Zentralbl Chir 9: 49-63, 1882 (In German).

8. Thompson LD and Heffner DK: The clinical importance of cystic squamous cell carcinomas in the neck: A study of 136 cases. Cancer 82: 944-956, 1998.

9. Goldenberg D, Sciubba J and Koch WM: Cystic metastasis from head and neck squamous cell cancer: A distinct disease variant? Head Neck 28: 633-638, 2006.

10. Yasui T, Morii E, Yamamoto Y, Yoshii T, Takenaka Y, Nakahara S, Todo T and Inohara H: Human papillomavirus and cystic node metastasis in oropharyngeal cancer and cancer of unknown primary origin. PLoS One 9: e95364, 2014.

11. Ang KK, Harris J, Wheeler R, Weber R, Rosenthal DI, Nguyen-Tân PF, Westra WH, Chung CH, Jordan RC, Lu C, et al: Human papillomavirus and survival of patients with oropharyngeal cancer. N Engl J Med 363: 24-35, 2010. 